\title{
Evaluation of Privacy Preserving Algorithms Using Traffic Knowledge Based Adversary Models
}

\author{
Zhanbo Sun, Bin Zan*, Jeff (Xuegang) Ban, Marco Gruteser* and Peng Hao \\ Rensselaer Polytechnic Institute \\ 110 8th St. Troy, NY 12180-3590 \\ \{sunz2, banx, haop\}@rpi.edu \\ *WINLAB, Rutgers University \\ *671 Route 1 South, North Brunswick, NJ 08902-3390 \\ *\{zanb, gruteser\}@winlab.rutgers.edu
}

\begin{abstract}
By providing location traces of individual vehicles, mobile traffic sensors have quickly emerged as an important data source for traffic applications. In dealing with the privacy issues associated with this, researchers have been proposing different privacy protection algorithms. In this paper, we propose traffic-knowledge-based adversary models to attack privacy algorithms. By doing so, we can compare and evaluate different privacy algorithms in terms of both privacy protection and the convenience for traffic modeling. Results show that by having a relatively good privacy performance, the released datasets of both the 3.3 level of confusion entropy and the 0.1 individual likelihood can still be applied for a fine level of traffic applications.
\end{abstract}

\section{INTRODUCTION}

$\mathrm{M}$ OBILE traffic sensors - those move with the traffic flow and have the potential to track the movement of individual vehicles by using location traces - have quickly emerged as an important data source and been widely used. Meanwhile, there are always privacy concerns associated with this approach [4], [7]. Some proposed privacy preserving methods try to address this issue via naïve anonymization techniques which simply remove vehicle identifiers [12]. However, by linking the driving pattern and sensitivity locations (e.g., home end, office building, etc.) with the driver, location traces can be easily re-identified. Other methods try to protect privacy by perturbing or reducing the accuracy for either spatial or temporal information [8], [11], [2]. In these cases, however, transportation modelers usually hesitate to use such datasets for traffic applications, especially for those requiring high accuracy spatial and temporal information (e.g., for arterial performance measurements). Similarly, reducing the sampling frequency [10] is also not a very promising approach, since the sampled location traces (say in a 1minute sampling interval) can barely be used for a fine level of traffic applications.

Hoh et al. (2007) [4] introduced a novel time-to confusion metric to evaluate group-wide privacy in a set of location traces. They then propose an uncertainty-aware path cloaking algorithm which yields the time-to-confusion

Manuscript received May 15, 2011. criterion, in which the uncertainty of tracking is measured by entropy. Hoh et al. (2008) [5] proposed the idea of Virtual Trip Line (VTL), and showed that by using VTLs to regulate location and speed reports, privacy violations can be reduced. On top of that, Zan et al. (2011) [6] proposed a VTL zone-based path cloaking algorithm. The algorithm predefines VTL zones over the intersections of interests and only those vehicle trajectories within VTL zones (which also need to satisfy the entropy criterion) can be released. Using the released datasets as input, the success rate of traffic applications (in this case, queue length estimation) is acceptable. These existing algorithms use purely statistical traffic knowledge; information such as path likelihood and travel time distributions is estimated using historical data. Interestingly enough, the link between two neighboring VTL zones is actually similar to the concept of mix-zone as proposed by Bereford and Stajano (2004) [1]. In fact, the VTL-zone based algorithm defines the areas where mobile data should be collected, which can be considered as the opposite way of the mix-zone algorithm that defines areas where data should be suppressed. We believe the VTL-zone concept, by focusing on where data should be collected, minimizes the data collection effort and can better satisfy the data needs for traffic applications. For more comparisons between VTL zone based path cloaking algorithm and mix zone approaches, one can refer to [6].

In this paper, we evaluate how resilient these algorithms are to adversaries with more sophisticated traffic knowledge. In particular, we consider knowledge of travel time in the mix zones and signal delay patterns. We then measure how effective these are in linking released vehicle trajectories from two VTL zones. Moreover, since entropy is not a very intuitive metric in the transportation community, we also present results in terms of tracking success probability. The algorithms are evaluated using microscopic traffic simulation. The results reveal that by having a relatively good privacy performance, the released datasets of both the 3.3 level of confusion entropy and the 0.1 individual likelihood can still be applied for a fine level of traffic applications.

The paper is comprised of 6 sections. In Section 2, we introduce several privacy algorithms. We then propose our traffic-knowledge-based adversary models in Section 3. 
Section 4 describes the performance measures we use to evaluate different privacy algorithms. Experiments and numerical results are shown in Section 5, followed by the conclusions in Section 6.

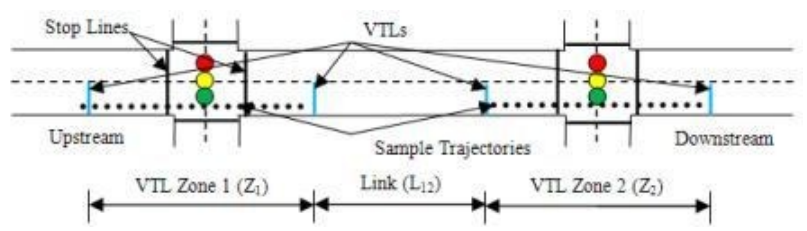

Fig. 1. VTL zones (Case 1)

\section{THE PRIVACY PRESERVING ALGORITHMS}

In this section, we introduce several privacy algorithms including the baseline case, random sampling, individual likelihood based and entropy based algorithms. Detailed discussions of some of those algorithms can be found in [6].

\section{A. VTL Zone and Baseline Case}

Being comprised of two Virtual Trip Lines (VTLs, [5]), one VTL zone covers one direction of an intersection. The two VTLs are designated to avoid major deceleration and acceleration processes due to traffic signals. GPS trace data is only collected within VTL zones: starting with the sample right before a vehicle enters into a VTL zone and ending with the sample right before a vehicle leaves a VTL zone. See Fig. 1. In this paper, the metric of privacy is based on the chance that vehicle trajectories may be linked at two VTL zones.

In the baseline case, all the vehicle trajectories within VTL zones are released. Because of the discontinuity of vehicle trajectories at the link between two VTL zones, it is not a trivial task for the adversary to keep tracking the vehicle trajectories between two VTL zones.

\section{B. Random Sampling}

On top of the baseline case, in order to enhance the level of privacy, a naïve random sampling approach is proposed. In which a proportion of the sample trajectories is randomly selected and released at each VTL zones, so that it is even harder for the adversary to continuously tracking vehicle trajectories between two VTL zones.

\section{Individual Likelihood}

Inspired by the individual likelihood of being tracked [6], we use equation (1) as a privacy metric, which illustrates the likelihood of taking a given time period for a particular vehicle to go from one VTL zone to another, normalized over the summation of the likelihoods of all the possible vehicles which cannot be distinguished from the object vehicle.

$$
p_{v \rightarrow c}^{d}=\frac{\rho_{v \rightarrow c^{*}} p_{T}\left(t_{v \rightarrow c}^{d}\right)}{\sum_{v^{\prime} \in V \backslash c} \sum_{d^{\prime} \in D_{v^{\prime}}} \rho_{v^{\prime} \rightarrow c^{*}} * p_{T}\left(t_{v^{\prime} \rightarrow c}^{d^{\prime}}\right)}
$$

Where $D$ is the whole set of vehicle IDs, $V$ is the whole set of VTL zones, $d$ is the target vehicle we are trying to track, which have the latest disclosed trajectory in VTL zone $v, c$ is the current VTL zone we are looking at, $\rho_{v \rightarrow c}$ is the likelihood of any vehicle go through the path from $v$ to $c$, which is calculated using historical data, $t_{v \rightarrow c}^{d}$ is the time period it takes for vehicle $d$ to go from VTL zone $v$ to $c$, and $p_{T}(\cdot)$ is a discrete probability density function of the travel time distribution (from $v$ to $c$ ), which yields a three parameter Log-normal distribution, parameterized by Least Square Estimation (LSE) using historical data.

Notice that the trajectory of vehicle $d$ at VTL zone $c$ cannot be released when the individual likelihood $p_{v \rightarrow c}^{d}$ is larger than a predefined level, e.g., 0.1.

\section{Entropy}

Some researchers have used entropy to measure the tracking uncertainty [4], [6] and equation (2) is proposed to calculate the system-wide tracking uncertainty.

$$
H=-\sum_{v \in V \backslash c} \sum_{d \in D_{v}}\left(p_{v \rightarrow c}^{d} \log p_{v \rightarrow c}^{d}\right)
$$

Where $H$ is the entropy metric and other related terms have already been defined in equation (1). By comparing the privacy metric $H$ with a predefined confusion level $\alpha$, we can then determine if one sample can be released or not. Intuitively, entropy is related to the factor that among how many vehicles the target vehicle can be indistinguishable. It is also an aggregated measure of the probability of not tracking vehicles between two VTL zones. For example, when the entropy is equal to 0.95 , this is about to say that one target vehicle is indistinguishable between two vehicles. We also try to combine both individual likelihood and entropy as the privacy metrics, and the overall performance has no major deviation from the one of individual likelihood dataset.

\section{TRAFFIC-KNOWLEDGE-BASED ADVERSARY MODEL}

In this section, we propose traffic-knowledge-based adversary models, which can be used to attack the released datasets by trying to link vehicle trajectories from two different VTL zones together. Two general cases are considered.

\section{A. Case 1}

Consider two neighboring VTL zones $\left(Z_{1}\right.$ and $\left.Z_{2}\right)$ which cover two consecutive intersections, and with one link $\left(L_{12}\right)$ in between these two VTL zones, as shown in Fig. 1. On $L_{12}$, since vehicles are usually proceeding at a speed close to the Free Flow Speed and the acceleration/deceleration processes are usually unnoticeable, the travel time on $L_{12}$ is very stable. In this paper, we propose two methods to estimate the travel time on $L_{12}$, one is simply the Free Flow Travel Time, which can be obtained by the length of $L_{12}$ divided by the design speed; the other is an adjusted travel time, which can be obtained by the length of $L_{12}$ divided by the estimated average speed on this link. The estimated average speed is calculated by taking the average of the speed of the last sample in $Z_{1}$ and the first sample in $Z_{2}$. In essence, the adversary does not know which 
travel time is closer to the ground truth; however, it is conservative to assume that the adversary will always choose the travel time which has the best performance, referred as $T_{12}$.

Now target on a set of released vehicles $\left(\Omega_{1}\right)$ which go through $Z_{1}$. Then for vehicle $n \epsilon \Omega_{1}$, based on its trajectory in $Z_{1}$, it is easy to tell when it leaves $Z_{1}$, referred as $T_{1}^{n}$. The time that $n$ enters $Z_{2}$ can then be approximated as $T_{1}^{n}+T_{12}^{n}$, referred as $T_{2}^{n}$. See equation (3).

$$
T_{2}^{n} \approx T_{1}^{n}+T_{12}^{n}
$$

If we slightly relax the travel time estimation and give a threshold $T_{t}$, then the vehicle enters VTL zone 2 within time period $\left[T_{2}^{n}-T_{t}, T_{2}^{n}+T_{t}\right]$ are very likely to be the same vehicle as vehicle $n$. In other words, consider a set of released vehicles $\left(\Omega_{2}\right)$ which go through $Z_{2}$, then for vehicle $m \epsilon \Omega_{2}$, with $T_{2}^{m}$ as the time that vehicle $m$ enters into $Z_{2}$, if $T_{2}^{n}-T_{t} \leq T_{2}^{m} \leq T_{2}^{n}+T_{t}$, we add $m$ into a suspect list $(S)$, meaning that $m$ is likely to be the same vehicle as $n$. If $S$ is not empty, we can then choose the vehicle $\widehat{k}$ which satisfies equation (4) as an inference.

$$
\widehat{k}=\operatorname{argmin}_{k \in S}\left|T_{2}^{k}-T_{2}^{n}\right|
$$

That means $\hat{k}$ is the vehicle whose entrance time to $Z_{2}$ is the closest to the estimated entrance time of vehicle $n\left(T_{2}^{n}\right)$. If the inference is correct $(\hat{k}=n)$, the vehicle trajectories at two neighboring VTL zones can be linked, the privacy is thus violated.

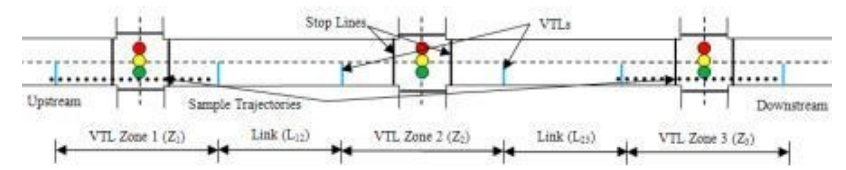

Fig. 2. VTL zones (Case 2)

\section{B. Case 2}

Consider two VTL zones which are not neighbored with each other (e.g., $Z_{1}, Z_{2}$ and $Z_{3}$, which cover a corridor with three intersections, and the adversary model is trying to link the vehicle trajectories from $Z_{1}$ to $Z_{3}$, as shown in Fig. 2). Following the same logic as Case 1, the travel times on the links $\left(L_{12}, L_{23}\right)$ between two consecutive VTL zones are very stable, which can be estimated as $T_{12}$ and $T_{23}$, using the same approaches as Case 1.

Now we attempt to link the trajectories from a set of released vehicles $\left(\Omega_{1}\right)$ which go through $Z_{1}$ to a set of released vehicles $\left(\Omega_{3}\right)$ which go through $Z_{3}$. For vehicle $n \epsilon \Omega_{1}$, the time that $n$ enters $Z_{3}$ can then be approximated as equation (5).

$$
T_{3}^{n} \approx T_{1}^{n}+T_{12}^{n}+T_{2, D}^{n}+T_{23}^{n}
$$

Where $T_{3}^{n}$ is the estimated time that $n$ enters $Z_{3}, T_{1}^{n}$ is the time that $n$ leaves $Z_{1} ; T_{12}^{n}$ and $T_{23}^{n}$ are the estimated travel time of $n$ on the links between $Z_{1}$ and $Z_{2}$, and between $Z_{2}$ and $Z_{3}$, respectively; $T_{2, D}^{n}$ is the travel time of vehicle $n$ within $Z_{2}$, which can be estimated via the delay pattern of $Z_{2}$

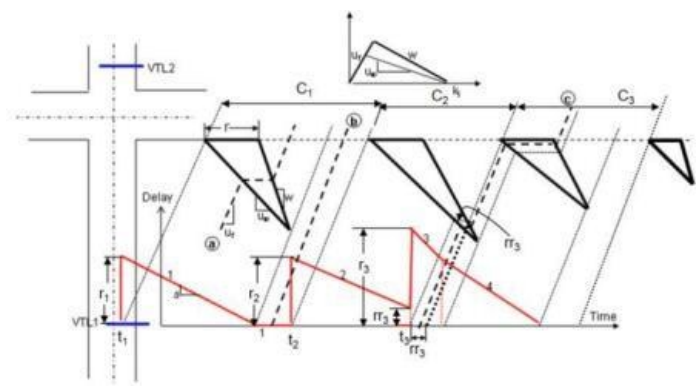

Fig. 3. Reconstruction delay pattern. A typical signalized intersection is shown here. Given the trajectories of a set of released vehicles $\Omega_{2}$ that go through $Z_{2}$ (dashed lines) and the signal timing information, we can use the bold solid triangles (in the upper part) to represent how queue forms and dissipates. By analyzing the geometry of the triangles, we can then construct the theoretical delay curve (piecewise linear curves at the bottom), which indicates the travel time an imaginary vehicle will experience when arriving at the intersection at a given time.

[14]. See Fig. 3. Notice that the delay pattern of $Z_{2}$ is reconstructed using the trajectories of a set of released vehicles $\left(\Omega_{2}\right)$ which go through $Z_{2}$, and the signal timing information. Thus for vehicle $m \in \Omega_{3}$, with $T_{3}^{m}$ as the time that vehicle $m$ enters into $Z_{3}$, if $T_{3}^{n}-T_{t} \leq T_{3}^{m} \leq T_{3}^{n}+T_{t}$, we add $m$ into a suspect list $(S)$. If $S$ is not empty, we can then choose the vehicle $\widehat{k}$ which satisfies equation (6) as an inference.

$$
\hat{k}=\operatorname{argmin}_{k \in S}\left|T_{3}^{k}-T_{3}^{n}\right|
$$

If the inference is correct $(\hat{k}=n)$, the vehicle trajectories at $Z_{1}$ and $Z_{3}$ can be linked, the privacy is thus violated.

\section{PERFORMANCE MEASURES}

In this section, we describe the measures we use to evaluate the performance of the privacy algorithms, with respect to both privacy protection and the convenience for traffic modeling.

\section{A. Privacy Protection}

In terms of privacy protection, the performance of the privacy models can be evaluated by applying the adversary models. In this paper, we use two measures to indicate this, namely, the percentage of tracked trajectories $\left(P_{1}\right)$ and the percentage of correct inference $\left(P_{2}\right)$. Obtained by using the number of correct inference divided by the total number of trajectories (do not necessarily need to be released) going through both the VTL zones (e.g., both $Z_{1}$ and $Z_{2}$ in Case $1 ; Z_{1}, Z_{2}$ and $Z_{3}$ in Case 2), $P_{1}$ indicates the probability that the trajectories of one vehicle can be successfully linked at the two VTL zones. Different from $P_{1}, P_{2}$ is obtained by using the number of correct inference divided by the total number of inference, which indicates how accurate the inferences are.

From the perspective of individuals, $P_{1}$ may seem to be more important, since it reveals the potential risk that one vehicle trajectory can be tracked. However, $P_{2}$ is indeed equally important, because even though the trajectories can be linked, the linkage is highly possible to be incorrect, thus the adversary has no way to prove that the linked trajectories 
correspond to the same driver.

Notice that in our adversary models, one released vehicle trajectory in the current VTL zone (e.g., $Z_{1}$, as of Case 1) can at most correspond to one inference (which is selected from the suspect list) based on equation (4) or (6). It is also worthy to mention that there are some situations in which the suspect list $(S)$ is empty, therefore no inference can be made in these situations.

\section{B. Convenience for Traffic Modeling}

A tradeoff usually exists between privacy protection and traffic applications, meaning by having a high level of privacy, transportation researchers may have to, to some extent, sacrifice the ease of traffic modeling. Thus it is important to make sure that after applying the privacy algorithms, the released datasets can still be used for traffic modeling, especially (and to the greatest interest of the author) arterial performance estimation (e.g., queue length estimation, delay pattern estimation, etc.). Two measures are used in our work: one is the released number of trajectories in each VTL zone (compared with the total number of trajectories in the baseline case); the other is the percentage of the number of cycles (out of the total number of cycles in the simulation) for which queue length estimation can be successfully performed, which is defined as the success rate in [13].

\section{EXPERIMENT AND NUMERICAL RESULTS}

In this section, we evaluate the privacy algorithms using the performance measures we mentioned in section IV. Detailed results of the numerical experiment are presented here.

\section{A. Simulation Settings}

The traffic simulation is run in Paramics for about an hour. Vehicle trajectory data is then extracted from a subnetwork of the SR41 corridor located at the city of Fresno, CA [9]. The selected network covers over 90 signalized intersection and 15 ramp metering controller. A total number of 102 VTL zones are deployed. See Fig. 4.

\section{B. Evaluation in Terms of Privacy Preserving}

By using the traffic-knowledge-based adversary model to attack the released datasets, we can evaluate the performance of the privacy algorithms in terms of privacy preserving.

\section{1) Case 1}

For Case 1, we list the results of 10 pairs of VTL zones, in which the traffic volumes are relatively large. Table 1 shows the performance of baseline case: column 2 and column 3 are the upstream and downstream VTL zones, expressed by the link sequence that one VTL zone covers; column 4 is the number of released trajectories which go through both $Z_{1}$ and $Z_{2}$ (as of the baseline case, trajectories of all vehicles are released); column 5 and column 6 are the number of released trajectories within $Z_{1}$ and $Z_{2}$, respectively; column 7 is the number of inferences can be made, notice that one released trajectory in $Z_{1}$ can at most correspond to one inference (it is

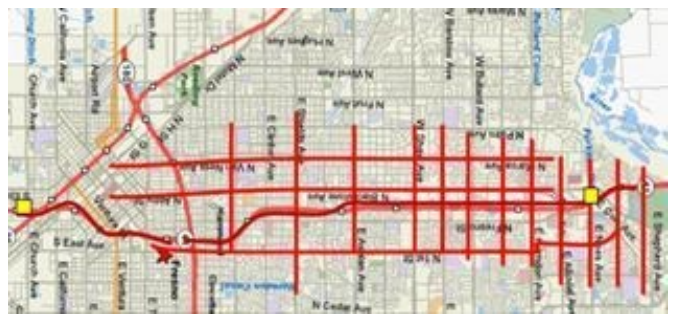

Fig. 4. Simulation network

possible that $S$ is empty, no inference can be made in this case); column 8 is the number of correct inferences; column 9 corresponds to $P_{1}$ in section IV, which can be obtained by using column 8 divided by the actual number of trajectories which go through both $Z_{1}$ and $Z_{2}$; column 10 corresponds to $P_{2}$ in section IV, which can be obtained by using column 8 divided by column 7 .

Table 1 essentially illustrates that the idea of only releasing vehicle trajectories within VTL zones is, to some extent, useful to preserve privacy; however, this may not be sufficient since there are still a large proportion (about 35\% in average) of vehicles trajectories can be successful tracked.

The results for random sampling $(50 \%$ trajectories are released) case are shown in Table 2. Compared with the baseline case, random sampling models are able to protect privacy by filtering out some sample trajectories at each VTL zone, so that fewer trajectories can be tracked and more inaccurate the inference becomes. As it is shown in Fig. 5, when sampling rate decreases, more trajectories are filtered out, and as a result, the first privacy measure $\left(P_{1}\right)$ drops. However, the second privacy measure $\left(P_{2}\right)$ may not necessarily decrease as the sampling rate increases, meaning the random sampling method cannot guarantee the accuracy of the inferences will decrease as more samples are filtered out, see Fig. 6.

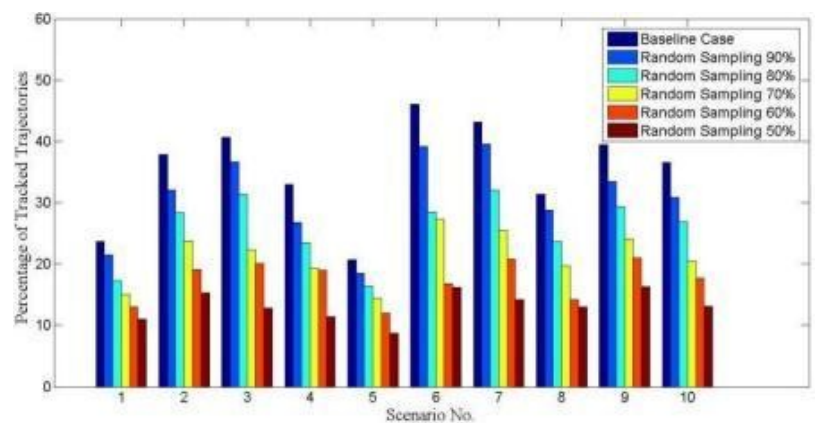

Fig. 5. Baseline case vs. random sampling $\left(P_{1}\right)$

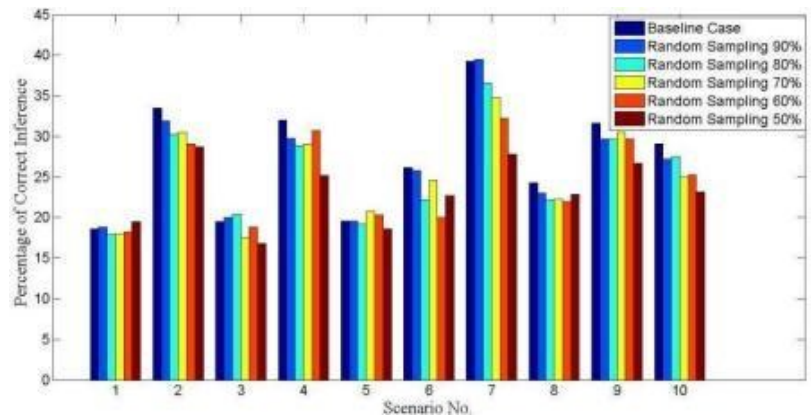

Fig. 6. Baseline case vs. random sampling $\left(P_{2}\right)$ 
Table 1. Privacy performance of the baseline dataset (Case 1)

\begin{tabular}{|c|c|c|c|c|c|c|c|c|c|}
\hline No. & $Z_{1}$ & $Z_{2}$ & $\begin{array}{c}\text { No. of Released } \\
\text { Trajectories } \\
\text { (Both } Z_{1} \text { and } \\
Z_{2} \text { ) }\end{array}$ & $\begin{array}{l}\text { No. of Released } \\
\text { Trajectories }\left(Z_{1}\right)\end{array}$ & $\begin{array}{c}\text { No. of } \\
\text { Released } \\
\text { Trajectories } \\
\left(Z_{2}\right)\end{array}$ & $\begin{array}{c}\text { No. of } \\
\text { Inferences }\end{array}$ & $\begin{array}{c}\text { No. of } \\
\text { Correct } \\
\text { Inferences }\end{array}$ & $\begin{array}{l}\text { Percentage of } \\
\text { Tracked } \\
\text { Trajectories }\end{array}$ & $\begin{array}{l}\text { Percentage of } \\
\text { Correct } \\
\text { Inferences }\end{array}$ \\
\hline 1 & 597_598_599 & 601_602 & 1136 & 1496 & 1225 & 1446 & 269 & $23.7 \%$ & $18.6 \%$ \\
\hline 2 & 554555 & 556557 & 517 & 586 & 1144 & 585 & 196 & $37.9 \%$ & $33.5 \%$ \\
\hline 3 & $556 \_557$ & $559 \_560$ & 452 & 1144 & 622 & 944 & 184 & $40.7 \%$ & $19.5 \%$ \\
\hline 4 & $559 \quad 560$ & $562 \quad 563$ & 579 & 622 & 757 & 597 & 191 & $33.0 \%$ & $32.0 \%$ \\
\hline 5 & 612_613_614 & 616_617 & 886 & 969 & 1112 & 934 & 183 & $20.7 \%$ & $19.6 \%$ \\
\hline 6 & $658 \quad 659$ & $\begin{array}{lll}660 & 661 & 662\end{array}$ & 495 & 999 & 642 & 871 & 228 & $46.1 \%$ & $26.2 \%$ \\
\hline 7 & 780_781_782 & $784 \_785$ & 424 & 475 & 923 & 466 & 183 & $43.2 \%$ & $39.3 \%$ \\
\hline 8 & $690 \_691 \_692$ & $692 \_693 \_694$ & 625 & 915 & 672 & 807 & 196 & $31.4 \%$ & $24.3 \%$ \\
\hline 9 & 692_693_694 & 694_695_696 & 514 & 672 & 599 & 643 & 203 & $39.5 \%$ & $31.6 \%$ \\
\hline 10 & $529 \_530$ & 531_532_533 & 673 & 892 & 765 & 846 & 246 & $36.6 \%$ & $29.1 \%$ \\
\hline
\end{tabular}

Table 2. Privacy performance of the $50 \%$ random sampling dataset (Case 1)

\begin{tabular}{|c|c|c|c|c|c|c|c|c|c|}
\hline No. & $Z_{1}$ & $Z_{2}$ & $\begin{array}{l}\text { No. of Released } \\
\text { Trajectories } \\
\text { (Both } Z_{1} \text { and } Z_{2} \text { ) }\end{array}$ & $\begin{array}{l}\text { No. of Released } \\
\text { Trajectories }\left(Z_{1}\right)\end{array}$ & $\begin{array}{l}\text { No. of Released } \\
\text { Trajectories }\left(Z_{2}\right)\end{array}$ & $\begin{array}{c}\text { No. of } \\
\text { Inferences }\end{array}$ & $\begin{array}{c}\text { No. of } \\
\text { Correct } \\
\text { Inferences }\end{array}$ & $\begin{array}{l}\text { Percentage of } \\
\text { Tracked } \\
\text { Trajectories }\end{array}$ & $\begin{array}{l}\text { Percentage } \\
\text { of Correct } \\
\text { Inferences }\end{array}$ \\
\hline 1 & $\begin{array}{llll}597 \quad 598 \quad 599\end{array}$ & $601 \quad 602$ & 278 & 729 & 604 & 640 & 125 & $11.0 \%$ & $19.5 \%$ \\
\hline 2 & $5 \overline{54} 5 \overline{55}$ & 556557 & 136 & 309 & 564 & 275 & 79 & $15.3 \%$ & $28.7 \%$ \\
\hline 3 & $556 \_557$ & $559 \_560$ & 104 & 564 & 306 & 345 & 58 & $12.8 \%$ & $16.8 \%$ \\
\hline 4 & 559560 & 562563 & 136 & 306 & 380 & 262 & 66 & $11.4 \%$ & $25.2 \%$ \\
\hline 5 & 612_613_614 & 616_617 & 231 & 497 & 559 & 413 & 77 & $8.7 \%$ & $18.6 \%$ \\
\hline 6 & $658 \quad 659$ & $660 \_661 \_662$ & 135 & 510 & 314 & 353 & 80 & $16.2 \%$ & $22.7 \%$ \\
\hline 7 & $780 \quad 781 \quad 782$ & 784785 & 97 & 237 & 443 & 216 & 60 & $14.2 \%$ & $27.8 \%$ \\
\hline 8 & $690 \quad 691 \quad 692$ & $\begin{array}{lll}692 & 693 & 694\end{array}$ & 169 & 459 & 362 & 353 & 81 & $13.0 \%$ & $22.9 \%$ \\
\hline 9 & $692 \_693 \_694$ & 694_695_696 & 140 & 362 & 299 & 315 & 84 & $16.3 \%$ & $26.7 \%$ \\
\hline 10 & 529530 & $531 \quad 532 \quad 533$ & 172 & 427 & 389 & 380 & 88 & $13.1 \%$ & $23.2 \%$ \\
\hline
\end{tabular}

Table 3. Privacy performance of the 0.1 individual likelihood dataset (Case 1)

\begin{tabular}{|c|c|c|c|c|c|c|c|c|c|}
\hline No. & $Z_{1}$ & $Z_{2}$ & $\begin{array}{l}\text { No. of Released } \\
\text { Trajectories } \\
\text { (Both } Z_{1} \text { and } Z_{2} \text { ) }\end{array}$ & $\begin{array}{l}\text { No. of Released } \\
\text { Trajectories }\left(Z_{1}\right)\end{array}$ & $\begin{array}{l}\text { No. of Released } \\
\text { Trajectories }\left(Z_{2}\right)\end{array}$ & $\begin{array}{l}\text { No. of } \\
\text { Inferences }\end{array}$ & $\begin{array}{c}\text { No. of } \\
\text { Correct } \\
\text { Inferences }\end{array}$ & $\begin{array}{l}\text { Percentage of } \\
\text { Tracked } \\
\text { Trajectories }\end{array}$ & $\begin{array}{l}\text { Percentage } \\
\text { of Correct } \\
\text { Inferences }\end{array}$ \\
\hline 1 & 597_598_599 & $601 \_602$ & 341 & 1481 & 440 & 890 & 95 & $8.4 \%$ & $10.7 \%$ \\
\hline 2 & $5 \overline{54} \_555$ & $556 \_557$ & 177 & 519 & 870 & 412 & 75 & $14.5 \%$ & $18.2 \%$ \\
\hline 3 & $556 \_557$ & $559 \_560$ & 88 & 870 & 343 & 407 & 21 & $4.6 \%$ & $5.2 \%$ \\
\hline 4 & $559 \_560$ & $562 \_563$ & 36 & 343 & 466 & 262 & 11 & $1.9 \%$ & $4.2 \%$ \\
\hline 5 & 612_613_614 & $616 \quad 617$ & 227 & 560 & 673 & 462 & 76 & $8.6 \%$ & $16.5 \%$ \\
\hline 6 & $658 \quad 659$ & $660 \quad 661662$ & 51 & 468 & 413 & 266 & 11 & $2.2 \%$ & $4.1 \%$ \\
\hline 7 & $780 \quad 781 \quad 782$ & $7 \overline{784} 7 \overline{\overline{785}}$ & 91 & 334 & 709 & 296 & 58 & $13.7 \%$ & $19.6 \%$ \\
\hline 8 & 690_691_692 & 692_693_694 & 159 & 669 & 307 & 387 & 60 & $9.6 \%$ & $15.5 \%$ \\
\hline 9 & $692 \_693 \_694$ & 694_695_696 & 90 & 307 & 401 & 261 & 60 & $11.7 \%$ & $23.0 \%$ \\
\hline 10 & $529 \_530$ & 531_532_533 & 292 & 750 & 480 & 613 & 109 & $16.2 \%$ & $17.8 \%$ \\
\hline
\end{tabular}

Table 4. Privacy performance of the 3.3 level of confusion entropy dataset (Case 1)

\begin{tabular}{|c|c|c|c|c|c|c|c|c|c|}
\hline No. & $Z_{1}$ & $Z_{2}$ & $\begin{array}{l}\text { No. of Released } \\
\text { Trajectories } \\
\text { (Both } Z_{1} \text { and } Z_{2} \text { ) }\end{array}$ & $\begin{array}{l}\text { No. of Released } \\
\text { Trajectories }\left(Z_{1}\right)\end{array}$ & $\begin{array}{l}\text { No. of Released } \\
\text { Trajectories }\left(Z_{2}\right)\end{array}$ & $\begin{array}{l}\text { No. of } \\
\text { Inferences }\end{array}$ & $\begin{array}{c}\text { No. of } \\
\text { Correct } \\
\text { Inferences }\end{array}$ & $\begin{array}{c}\text { Percentage of } \\
\text { Tracked } \\
\text { Trajectories }\end{array}$ & $\begin{array}{l}\text { Percentage } \\
\text { of Correct } \\
\text { Inferences }\end{array}$ \\
\hline 1 & 597_598_599 & $601 \_602$ & 622 & 1492 & 709 & 1214 & 185 & $16.3 \%$ & $15.2 \%$ \\
\hline 2 & $554 \_555$ & 556_557 & 150 & 570 & 778 & 396 & 84 & $16.2 \%$ & $21.2 \%$ \\
\hline 3 & $556 \_557$ & $559 \_560$ & 174 & 778 & 461 & 414 & 66 & $14.6 \%$ & $15.9 \%$ \\
\hline 4 & $559 \_560$ & $562 \_563$ & 41 & 461 & 286 & 172 & 30 & $5.2 \%$ & $17.4 \%$ \\
\hline 5 & 612_613_614 & $616 \_617$ & 125 & 496 & 574 & 307 & 46 & $5.2 \%$ & $15.0 \%$ \\
\hline 6 & $658 \_659$ & $660 \_661 \_662$ & 30 & 385 & 364 & 118 & 13 & $2.6 \%$ & $11.0 \%$ \\
\hline 7 & 780_781_782 & $784 \_785$ & 28 & 345 & 550 & 93 & 18 & $4.2 \%$ & $19.4 \%$ \\
\hline 8 & 690_691_692 & 692_693_694 & 100 & 505 & 276 & 172 & 43 & $6.9 \%$ & $25.0 \%$ \\
\hline 9 & 692_693_694 & 694_695_696 & 80 & 276 & 363 & 209 & 51 & $9.9 \%$ & $24.4 \%$ \\
\hline 10 & $529 \_530$ & 531_532_533 & 226 & 583 & 478 & 488 & 107 & $15.9 \%$ & $21.9 \%$ \\
\hline
\end{tabular}




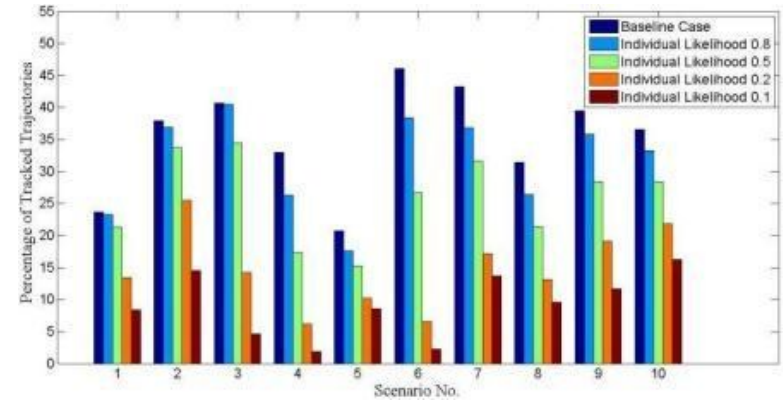

Fig. 7. Baseline case vs. individual likelihood $\left(P_{1}\right)$

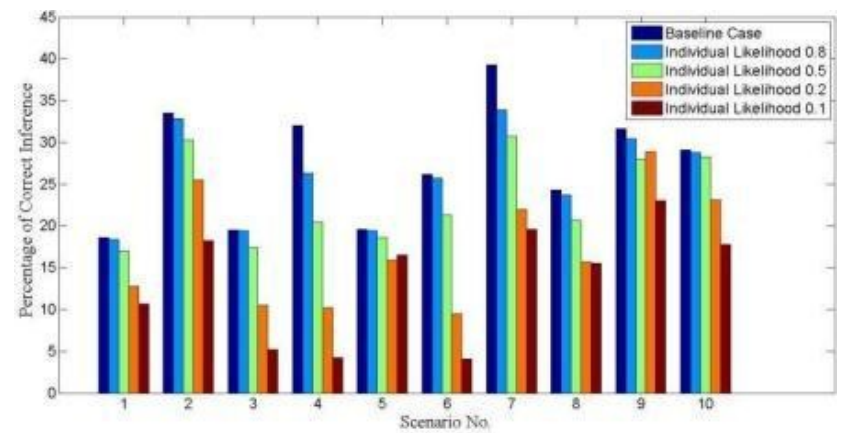

Fig. 8. Baseline case vs. individual likelihood $\left(P_{2}\right)$

Table 3 indicates the results of 0.1 individual likelihood dataset. By compared with Table 2, one can tell that while releasing much more samples than the random sampling dataset (e.g., Scenario No. 1, 2) or almost the same number of samples at each VTL zone (e.g. Scenario No. 5, 8), the individual likelihood dataset has overall higher level of privacy.

We also show the results for different individual likelihoods (e.g., 0.2, 0.5, 0.8), as shown in Fig. 7 and Fig. 8. In contrast to the random sampling method, a stricter threshold here leads to decreases in both $P_{1}$ and $P_{2}$, resulting a higher level of privacy.

The results for the 3.3 level of confusion entropy dataset are shown in Table 4. By comparing this with Table 3, one can find that in terms of privacy protection, these two datasets are comparable with each other, at least under the attack models considered here. For $P_{1}, 0.1$ individual likelihood dataset has better performance in 5 out of 10 examples; for $P_{2}, 0.1$ individual likelihood dataset has better performance in 8 out of 10 examples. As expected, a higher level of privacy can be obtained by using a higher level of confusion, as shown in Fig. 9 and Fig. 10.

Fig. 11 and Fig. 12 compare the performance of datasets generated by different privacy algorithms. Notice that the amounts of sample trajectories these three datasets release at each VTL zone are generally comparable. In terms of $P_{1}$, the performance of these three datasets are very close to each other, the 0.1 individual likelihood and the 3.3 level of confusion entropy dataset are slightly better than $50 \%$ random sampling dataset; in terms of $P_{2}, 0.1$ individual likelihood dataset has the best performance, followed by the 3.3 level of confusion entropy dataset and then the $50 \%$ random sampling dataset.

2) Case 2

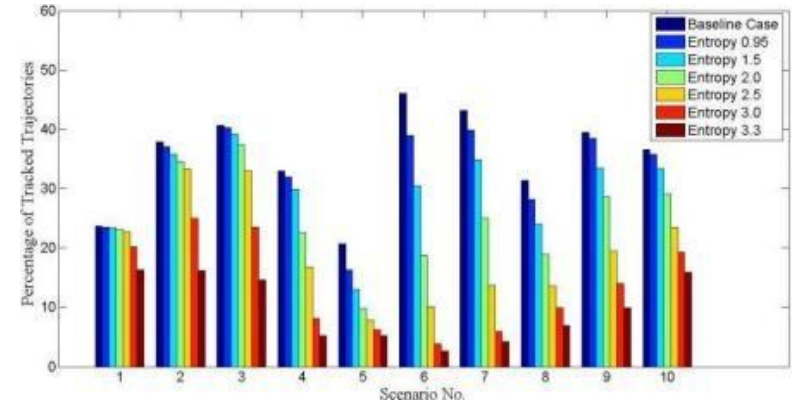

Fig. 9. Baseline case vs. entropy $\left(P_{1}\right)$

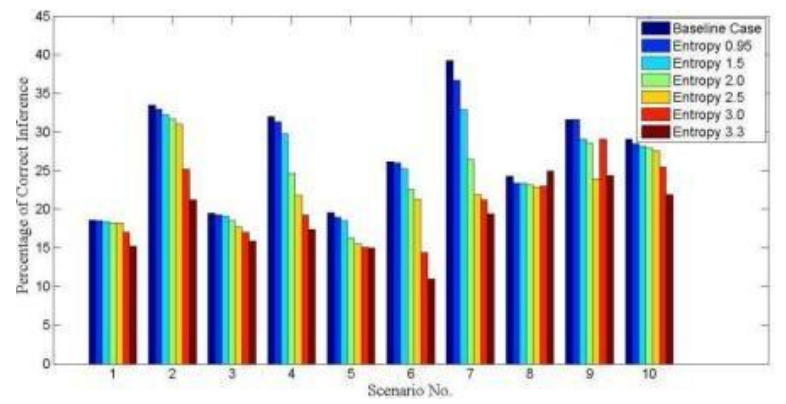

Fig. 10. Baseline case vs. entropy $\left(P_{2}\right)$

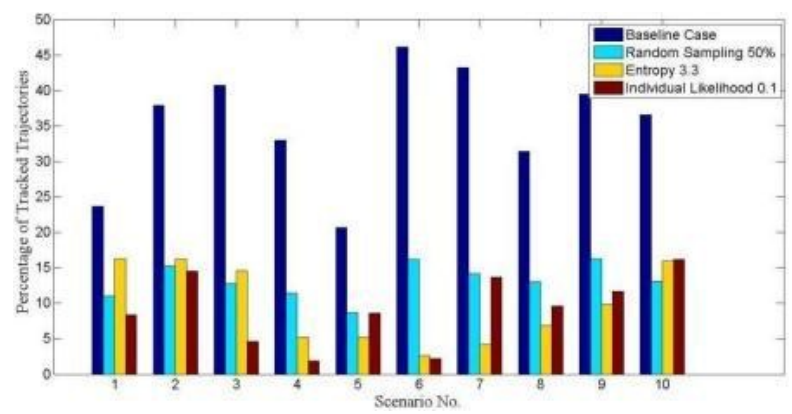

Fig. 11. Privacy performance of different datasets $\left(P_{1}\right)$

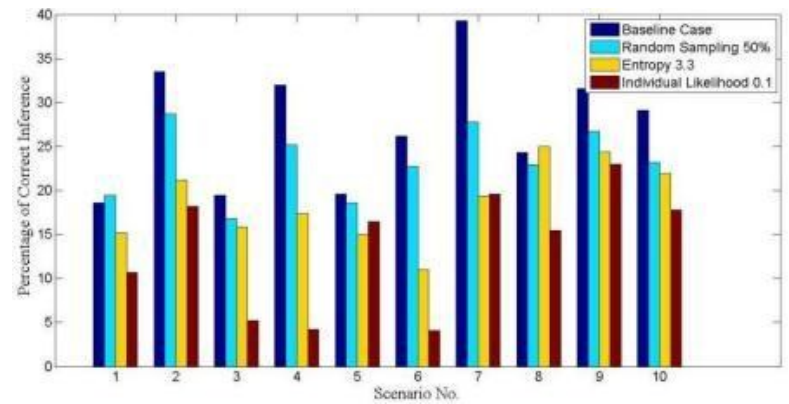

Fig. 12. Privacy performance of different datasets $\left(P_{2}\right)$

For case 2, here we list the results for 5 pairs of nonneighboring VTL zones. Table 5 shows that for the baseline case, the average percentage of tracked vehicles is about $12.6 \%$, and among all the inferences, about $6.8 \%$ in average are correct. This implies that even though the adversary has access to the signal information and a relatively good trafficknowledge-based travel time estimation model, it is still very difficult to successfully track vehicle trajectories from nonneighboring VTL zones.

Similar experiments have been done using random sampling datasets, individual likelihood datasets and entropy datasets. The results and implications generally match with 
Table 5. Privacy performance of the baseline dataset (Case 2)

\begin{tabular}{|c|c|c|c|c|c|c|c|c|c|}
\hline No. & $Z_{1}$ & $Z_{3}$ & $\begin{array}{l}\text { No. of Released } \\
\text { Trajectories } \\
\text { (Both } Z_{1} \text { and } Z_{3} \text { ) }\end{array}$ & $\begin{array}{l}\text { No. of Released } \\
\text { Trajectories }\left(Z_{1}\right)\end{array}$ & $\begin{array}{l}\text { No. of Released } \\
\text { Trajectories }\left(Z_{3}\right)\end{array}$ & $\begin{array}{c}\text { No. of } \\
\text { Inferences }\end{array}$ & $\begin{array}{c}\text { No. of } \\
\text { Correct } \\
\text { Inferences }\end{array}$ & $\begin{array}{l}\text { Percentage } \\
\text { of Tracked } \\
\text { Trajectories }\end{array}$ & $\begin{array}{l}\text { Percentage of } \\
\text { Correct } \\
\text { Inferences }\end{array}$ \\
\hline 1 & $554 \_555$ & $559 \quad 560$ & 197 & 586 & 622 & 495 & 39 & $19.8 \%$ & $7.9 \%$ \\
\hline 2 & $556 \_557$ & $562 \_563$ & 417 & 1144 & 757 & 972 & 60 & $14.4 \%$ & $6.2 \%$ \\
\hline 3 & $690 \_691 \_692$ & $694 \_695 \_696$ & 467 & 915 & 599 & 764 & 59 & $12.6 \%$ & $7.7 \%$ \\
\hline 4 & $529 \_530$ & $533 \_534 \_535$ & 553 & 892 & 730 & 651 & 55 & $9.9 \%$ & $8.4 \%$ \\
\hline 5 & 797_798 & $802 \_803 \_804$ & 185 & 749 & 341 & 317 & 12 & $6.5 \%$ & $3.8 \%$ \\
\hline
\end{tabular}

Table 6. Entropy vs. individual likelihood (convenience of traffic modeling)

\begin{tabular}{|c|c|c|c|c|c|c|c|}
\hline \multirow[b]{2}{*}{ No. } & \multirow[b]{2}{*}{ Intersection } & \multicolumn{2}{|c|}{ No. of Released Trajectories } & \multicolumn{3}{|c|}{ Success Rate for Queue Length Estimation } & \multirow[b]{2}{*}{ Winner } \\
\hline & & $\begin{array}{l}3.3 \text { level of } \\
\text { confusion }\end{array}$ & $\begin{array}{c}0.1 \text { individual } \\
\text { likelihood }\end{array}$ & $\begin{array}{l}3.3 \text { level of } \\
\text { confusion }\end{array}$ & $\begin{array}{c}0.1 \text { individual } \\
\text { likelihood }\end{array}$ & Baseline & \\
\hline 1 & 597 598_599 & $1492 / 1496$ & $1481 / 1496$ & $79.6 \%$ & $79.6 \%$ & $81.8 \%$ & Entropy \\
\hline 2 & 601_602 & $709 / 1225$ & $440 / 1225$ & $64.4 \%$ & $57.8 \%$ & $75.6 \%$ & Entropy \\
\hline 3 & $554 \quad 555$ & $570 / 586$ & $519 / 586$ & $96.4 \%$ & $96.4 \%$ & $96.4 \%$ & Entropy \\
\hline 4 & $556 \_557$ & $778 / 1144$ & $870 / 1144$ & $64.8 \%$ & $64.8 \%$ & $70.1 \%$ & Individual likelihood \\
\hline 5 & 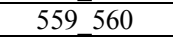 & $461 / 622$ & $343 / 622$ & $79.0 \%$ & $84.2 \%$ & $92.1 \%$ & Individual likelihood \\
\hline 6 & $562 \_563$ & $286 / 757$ & $466 / 757$ & $23.3 \%$ & $31.4 \%$ & $34.9 \%$ & Individual likelihood \\
\hline 7 & 612_613_614 & $496 / 969$ & $560 / 969$ & $65.2 \%$ & $69.6 \%$ & $91.3 \%$ & Individual likelihood \\
\hline 8 & $616 \_617$ & $574 / 1112$ & $673 / 1112$ & $76.7 \%$ & $83.7 \%$ & $88.4 \%$ & Individual likelihood \\
\hline 9 & $658 \_659$ & $385 / 999$ & $468 / 999$ & $40.2 \%$ & $45.7 \%$ & $66.3 \%$ & Individual likelihood \\
\hline 10 & 660_661_662 & $364 / 642$ & $413 / 642$ & $89.3 \%$ & $89.3 \%$ & $92.9 \%$ & Individual likelihood \\
\hline 11 & $780 \_781 \_782$ & $345 / 475$ & $334 / 475$ & $96.9 \%$ & $96.9 \%$ & $96.9 \%$ & Entropy \\
\hline 12 & $784 \quad 785$ & $550 / 923$ & $709 / 923$ & $64.9 \%$ & $71.9 \%$ & $76.2 \%$ & Individual likelihood \\
\hline 13 & 690_691_692 & $505 / 915$ & $669 / 915$ & $69.2 \%$ & $71.2 \%$ & $71.2 \%$ & Individual likelihood \\
\hline 14 & $692 \_693 \_694$ & $276 / 672$ & $307 / 672$ & $57.1 \%$ & $64.3 \%$ & $80.1 \%$ & Individual likelihood \\
\hline 15 & 694_695_696 & $363 / 599$ & $401 / 599$ & $36.9 \%$ & $35.4 \%$ & $38.5 \%$ & Entropy \\
\hline 16 & $529 \_530$ & $583 / 892$ & $750 / 892$ & $58.2 \%$ & $59.1 \%$ & $59.1 \%$ & Individual likelihood \\
\hline 17 & $531 \_532 \_533$ & $478 / 765$ & $480 / 765$ & $57.0 \%$ & $55.9 \%$ & $66.7 \%$ & Entropy \\
\hline
\end{tabular}

those of Case 1. It is worthy to mention that the level of privacy of baseline dataset is already quite high. Even though further implementation of other privacy algorithm can improve the performance, not too much improvement can be obtained. For example, for 0.1 individual likelihood dataset, the value of $P_{1}$ is $9.1 \%$ in average; and the value of $P_{2}$ is $7.8 \%$, which do not improve much from the baseline dataset.

\section{Evaluation in Terms of Modeling Convenience}

On top of an acceptable level of privacy, the modeling convenience of a released dataset should also be evaluated. Fig. 13 indicates the vehicle trajectories for the baseline dataset and Fig. 14 indicates the vehicle trajectories for the 0.1 individual likelihood dataset. As we can tell, in order to protect privacy, some of the trajectories have been filtered out in Fig. 14. And we want to test if the remaining trajectories are still enough for queue length estimation [13].

In particular, we compared 0.1 individual likelihood dataset and 3.3 level of confusion dataset, and the results are shown in Table 6. Notice that both datasets release almost all the trajectories at the first intersection. The reason for this is that this intersection is the very first intersection which is close to the entrance of the road network, thus both algorithms pretty much release all the trajectories at this intersection. For the cases where success rates are relatively low (e.g., about 30\% for intersection 6 and about 37\% for intersection 15), that is because some of the cycles for those intersections are uncongested, thus there is insufficient data (even for the baseline case) to support queue length

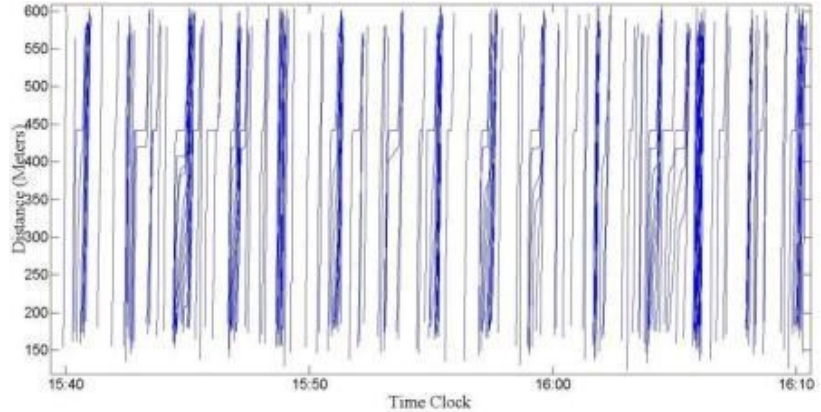

Fig. 13. Released vehicle trajectories (baseline case, 556_557)

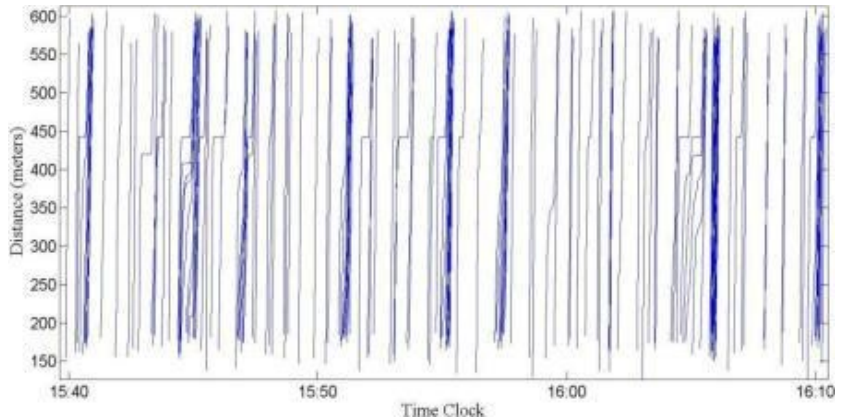

Fig. 14. Released vehicle trajectories (0.1 individual likelihood, 556_557)

estimation. There are also some cases in which one dataset releases more trajectories but ends up having a smaller success rate (e.g., intersection 5, intersection 15 and intersection 17). That is because the algorithm is releasing more samples in the cycles which already have many samples, but is not releasing enough samples in some cycles which have little samples. Compared with the baseline case, 
the success rate of both entropy dataset and individual likelihood dataset do not decrease much, implying that by filtering out some sample trajectories (to protect privacy), the remaining samples can still be applied for traffic applications. Moreover, one can find that in 11 out 17 cases, the 0.1 individual likelihood dataset has better performance than the entropy dataset with 3.3 level of confusion, which suggests that the individual likelihood datasets are more preferable in terms of the convenience for traffic applications.

\section{CONCLUSIONS}

In this paper, we developed traffic-knowledge-based adversary models to link vehicle trajectories between two different VTL zones. By applying this to different datasets, we can evaluate the performance of different privacy algorithms in terms of both privacy protection and the convenience for traffic modeling. It is found that the idea of only releasing trajectory data within VTL zones helps to protect privacy. And by comparing the performance of different privacy algorithms, we can conclude that privacy algorithms, especially those based on the metrics of individual likelihood and entropy, can enhance the level of privacy. Meanwhile, the released datasets of these algorithms can still be applied to traffic applications with satisfactory performances.

\section{ACKNOWLEDGMENT}

This research is supported by the National Science Foundation grant CMMI-1031452.

\section{REFERENCES}

[1] A. Beresford and F. Stajano, "Mix zones: user privacy in locationaware services," in proceedings of the second IEEE Annual Conference on Pervasive Computing and Communication Workshops, 2004.

[2] B. Gedik and L. Liu, "Location privacy in mobile systems: A personalized anonymization model," in distributed Computing Systems, 2005, ICDCS 2005, in proceedings. 25th IEEE International Conference on, 2005, pp. 620-629.

[3] B. Hoh and M. Gruteser, "Protecting location privacy through path confusion," in proceedings of IEEE/Create-Net SecureComm, Athens, Greece, September 2005.

[4] B. Hoh and M. Gruteser, "Preserving privacy in GPS traces via uncertainty-aware path cloaking," in proceedings of ACM CCS 2007, 2007.

[5] B. Hoh, M. Gruteser, R. Herring, J. Ban, D. Work, J.-C. Herrera, A. M. Bayen, M. Annavaram, and Q. Jacobson, "Virtual trip lines for distributed privacy-preserving traffic monitoring," in proceeding of the 6th international conference on Mobile systems, applications, and services, ser. MobiSys '08. New York, NY, USA: ACM, 2008, pp. 528. [Online]. Available: http://doi.acm.org/10.1145/1378600.1378604

[6] B. Zan, P. Hao, M. Gruteser, X., Ban, "VTL zone-based path cloaking algorithm," submitted to IEEE ITS Conference, 2011.

[7] F. Dotzer, "Privacy issues in vehicular ad hoc networks," in proceeding of the 2nd ACM international workshop on Vehicular ad hoc networks. ACM Press, 2005.

[8] H. Kargupta, S. Datta, Q. Wang, and K. Sivakumar, "Random data perturbation techniques and privacy preserving data mining," in IEEE ICDM. IEEE Press, 2003.

[9] H. Liu and S. Jabari, "Evaluation of corridor traffic management and planning strategies using microsimulation: a case study," Transportation Research Record, 2088, 2008, pp. 26-35.
[10] K. P. Tang, P. Keyani, J. Fogarty, and J. I. Hong, "Putting people in their place: an anonymous and privacy-sensitive approach to collecting sensed data in location-based applications," in proceedings of CHI '06, 2006, pp. 93-102.

[11] M. Gruteser, and D. Grunwald, "Anonymous usage of location-based services through spatial and temporal cloaking," in proceedings of the First International Conference on Mobile Systems, Applications, and Services, 2003.

[12] S. Rass, S. Fuchs and M. Schaffer, "How to protect privacy in floating car data systems," in proceeding of the fifth ACM international workshop on VehiculAr Inter-NETworking, 2008.

[13] X. Ban, P. Hao, Z. Sun, "Real time queue length estimation for signalized intersections using sampled travel times," Transportation Research, Part C, in press, 2011.

[14] X. Ban, R. Herring, P. Hao, and A. Bayen, “ Delay pattern estimation for signalized intersections using sampled travel times," Transportation Research Record 2130, 2009, pp. 109-119. 